\title{
The introduction of modern physics: overcoming a deformed vision of science
}

\author{
D. Gil and J. Solbes, Department de Didàctica de les Ciències Experimentals, \\ Universitat de València, Spain
}

In this paper, we try to show initially that modem physics is usually introduced in high school curricula without reference to the difficulties of classical physics, simply juxtaposing the two paradigms or even mixing them up. As a result, serious misconceptions arise. We then present another way of introducing modern physics, based on a constructivist view of science learning, and give some results obtained with the new materials.

\section{Introduction}

Several authors (Gil 1983, Hodson 1985, 1988, Millar and Driver 1987) have shown that science teaching is giving pupils and students an incorrect and simplistic image of science. 'This is marked by empiricism, but with an absence of its most relevant aspects, such as hypothesis-forming or experimental design. In this approach, the contributions of the epistemology during the last three decades (e.g., by Popper, Feyerabend, Kuhn, Lakatos and Toulmin) are largely bypassed. In our opinion, there is another cause of misunderstanding of the nature of science arising from the usual science teaching: this is the simple accumulative way of introducing knowledge whilst ignoring the search for any coherence of science, i.e., ignoring the importance of paradigms which guide the construction of new knowledge until insurmountable difficulties produce a theoretical revolution and the emergence of a new paradigm. In fact, it has been shown repeatedly that classical physics is introduced without taking into account pupils' preconceptions, i.e., the ideas that have to be displaced in the teaching of classical physics (Viennot 1976, Posner et al. 1982, McDermott 1984, Gil and Carrascosa 1985, Hashewh 1986).

We shall try to show in this paper that modern physics is usually introduced by high school curricula without reference to the difficulties of classical physics, either juxtaposing both paradigms or even mixing them up. This gives rise to serious misconceptions. We shall then present another way of introducing modern physics; this is based on a constructivist view of science learning. Finally, we shall show some results obtained with the new approach.

\section{Modern physics in high school curricula: a critical analysis}

With the purpose of showing how modern physics is introduced at the high school level and what the underlying image of science is, we have recently analysed a sample 
- the non-linear character of the development of physics;

- the difficulties which originated the crisis of classical physics; and

- the deep conceptual differences between classical and modern physics.

As a result, modern physics is introduced in a very simplistic way which gives rise to serious errors (Lehrman 1982, Garcia-Castañeda 1985). In fact, high percentages of these texts (between 68 and $79 \%$ ):

- interpret Einstein's equation $\mathrm{E}=\mathrm{mc}^{2}$ as an expression of mass/energy transformations (this error has also been detected in us university texts [Lehrman 1982], in spite of the critical analysis already published [Warren 1976]);

- reduce the corpuscle/wave duality to only the undulatory or the corpuscular aspect;

- explain the quantum indetermination as a lack of precision of instruments or as a random consequence;

- give a simplistic view of elementary particles as ultimate 'bricks' of matter without internal structure.

We also administered a questionnaire to 63 practising high school teachers, asking them about criteria and ways of introducing modern physics. Only $12 \%$ of the replies received referred to the necessity of starting with the problems relating to classical physics or to giving stress to the differences between the classical and the modern physics paradigms (or to any other approach which did not involve a mere
'accumulative' introduction of modern physics).

Given the widespread use of the accumulative form of introducing modern physics, we must expect the pupils' understanding of the main features of the new paradigm to be very poor. In fact, a questionnaire given by us to 536 pupils aged 16-18 has shown that:

(a) between 85 and $93 \%$ of the pupils ignore the existence of a crisis in the development of classical physics and are not capable of mentioning a single problem associated with this crisis or a difference between modern and classical physics;

(b) between 83 and $93 \%$ of pupils have deep misunderstandings about questions such as the limit character of the speed of light, the particle/wave duality, etc.

Further analyses conducted by us suggest that this unsatisfactory state is largely attributable to very incorrect ways of introducing modern physics. This is a serious situation because it produces an incorrect understanding not only of modern physics, but also of classical physics, by not clarifying the limits of validity of the latter and the differences between both paradigms.

\section{A constructivist approach}

In our opinion, the results mentioned in the foregoing paragraph are not due to the special difficulty of modern physics, but mainly to an incorrect teaching orientation which also affects classical physics.

Research into pupils' difficulties in the meaningful learning of many physics concepts has shown that pupils possess alternative conceptual frameworks which

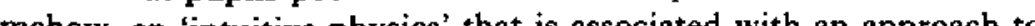
probems based ' an 'in accepted and generalized (Gil and Carrascosa 1985). The meaningful learning of the basic concepts of physics would demand not only a deep conceptual change (Posner et al. 1982) but also a methodological and epistemological change (Gil and Carrascosa 1985, 1990, Hashewh 1986) in the way of approaching problemsquestioning 'common-sense evidence', thinking in terms of hypotheses, etc.similar to that which took place with the construction of classical physics.

We could similarly suppose that pupils' difficulties in learning modern physics have an epistemological origin; that is to say, they come from an ignorance of the deep conceptual revolution that the emergence of the new paradigm constitutes. Any meaningful learning of the few elements of modern physics introduced in high school would then be obstructed by the linear, accumulative view presented. In brief: modern physics was constructed against the classical paradigm, and its meaningful learning would demand a similar approach.

It should, therefore, be relatively easy to obtain sensible improvements in pupils' learning, by using materials designed according to the constructivist model of science learning in a perspective of conceptual and methodological change. With this orientation, which takes into account both the pupils' conceptual frameworks and their methodological habits, it is possible to give a deeper and richer view of science and to make possible the conceptual changes which take place from 'common-sense physics' to the classical paradigm and from this to modern physics.

'This approach demands, in particular, that we replace the traditional teaching/ learning orientation, which places emphasis on the simple transmission/reception of knowledge, by a curriculum approach which involves the learners in activities and exposes them to problematical situations through which the knowledge can be (re)constructed by pupils (Driver and Oldham 1986, Hodson 1988, Wheatley 1991).

We shall describe in the next section an attempt at developing a suitable programme of activities for the introduction of modern physics, but first we shall set out very briefly some characteristics of what we call a programme of activities and the way of using this in the classroom (Gil et al. 1991).

The use of a programme of activities tries to favour collective work, by dividing the class into small groups. Each activity is worked on by each group, and the teacher then runs the interchanges between the groups and with the scientific community (represented by the teacher himself, the books and documents he can produce...). In this way, it is possible to stress the nature of science as a social construction (Solomon 1987, Wheatley 1991) without trivializing this construction: pupils must not believe, for instance, that an experiment done only by them can verify a hypothesis (Hodson 1985); the scientific community is, of course, much more exigent. But the teacher can help the small groups to place their work in the context of what scientists have done in more rigorous conditions.

It is not a question of displacing the transmission of knowledge by an impossible autonomous, isolated construction: in some sense the status of the small groups is that of 'novice researchers' working in a domain very well known by their 'lab director' (the teacher). It is the teacher (or better, the teachers' team) who prepares carefully the work to be done (the activities) and who reformulates and synthesizes the contribution of small groups, introducing, if necessary, other pieces of 
A programme of activities for the introduction of modern physics

According to the constructivist orientation that we have just summarized, we have designed a programme of activities to put pupils in a situation of (re)constructing knowledge and, at the same time, of getting acquainted with some characteristics of knowledge and, at the
the scientific approach.

This programme was conceived basically to show the limits of classical physics which constitutes, without any doubt, the core of the physics introduced in high schools. To this end, the programme begins with an activity which asks pupils to make a summary of classical physics in order to elicit the image of matter given by its main contributions (the powerful, coherent body of knowledge built up) and also some of the 'small' problems not solved by it. This review makes pupils remember that classical physics was constructed against another view, i.e. the 'common-sense physics' which had been incapable of solving many relevant problems. So, pupils are better prepared to understand that the difficulties appearing in the classical pradigm could - if not solved-originate a new crisis.

It is not possible to describe here the full programme (Solbes 1986) and we shall present, and comment on, only some activities, beginning with the four activities which constitute the introduction of the programme:

A.1. Review some of the main contributions (including its technical applications) of what is known as classical physics (i.e., the physics constructed from, let us say, Galileo to Maxwell)

A.2. Elicit the image of matter associated with classical physics (particularly which ideas about space, time, corpuscles and radiation are sustained).

A.3. Recognize that physics arose from what is known as 'common-sense physics' which could not solve certain relevant problems. Recognize, at the same time, that the theses of the pre-Galilean physics were displaced by the new classical paradigm.
A.4. Realize that classical physics managed to explain practically all the known A.4. Realize that classical physics managed to explain practically all the known phenomena and became a coherent body of knowledge which falled in

This last activity helps pupils to remember the problems (conveniently enhanced by the teacher when first encountered) posed by continuous and discontinuous spectra, the photoelectric effect, or the difficulties in proving the existence of a reference system in absolute rest. 'Thu study of this last problem - through new activities makes pupils conceive of experiments similar to those of Michelson and Morley. We propose then the analysis, by the pupils, of the results obtained in these experiments. This forces them to question the existence of an absolute space and an absolute timescale. It is convenient to point out the high methodological value of this critical analysis, done initially by Einstein, of the implicit assumptions of classical mechanics: implicit assumptions - because they are accepted as obvious, eluding any difficulties in the development of science. Once the relativity of time and space intervals for different observers are accepted as hypotheses, pupils can derive quite easily the variations of space, time and mass with the speed and the mass-energy relationship. It is necessary, of course, to avoid the
1982).

For the introduction of quantum physics, our programme starts with the difficulties in explaining the photoelectric effect: predictions based on Maxwell's conceptions of radiation are falsified by experiments. We propose, then, this activity:

Suggest a new hypothesis about the distribution of radiant energy to explain why - as we have seen - if the light frequency is above a certain value, it is possible to liberae frequency value, even with a very high light intensity sthat is to say, even if much light arrives at the surface)

This activity helps pupils to conceive the quantum hypothesis and makes it possible to obtain thereafter Einstein's equation for the photoelectric effect. We refer once ain to other pubishedn activities (Solbes 1986). Let us add that, after this ad hoc introduction of the activities (Solbes 1986). Let us add that, after this ad hoc introduction of the
quantum hypothesis, other activities help pupils to conceive De Broglie's hypothesis of the particle-wave duality of matter and reach a completely different view of the behaviour of matter (including the introduction of the uncertainty principle, associated with the wave character of matter).

The last activities are designed to review the most outstanding differences between the classical and the quantum views and to enhance the contributions of modern physics to the scientific and technical development of the 20 th century.

This programme of activities has been used with many high school students since 1982 , and an experimental study has recently been undertaken to verify the possibility of clear improvements in meaningful learning of modern physics.

To this end, one of the authors of this paper has taught 180 pupils (aged 16-18), using the complete programme of activities elaborated for the introduction of modern physics. Two other teachers have used a shorter version of the programme in order to see whether, even with brief utilization, the new teaching orientation could produce a clear improvement in pupils' understanding. A full description of this experiment has been given by Solbes (1986).

\section{Some results}

The results obtained with the experimental groups show, as expected, a very clear improvement of pupils' understanding. Only a small percentage (between 8 and $31 \%$ ) of the pupils who were exposed to the programme of activities ignored - at the end of the course - the existence of a crisis in the development of physics and some of the problems which caused the crisis of classical physics, or any difference between classical and modern physics. Even with a shortened version of the programme of activities, the percentages remained at between 26 and $45 \%$, whereas for nonexperimental groups these percentages reached between 85 and $94 \%$.

Of the pupils exposed to the programme of activities, only one-third showed misconceptions about the mass/energy relationship, the particle/wave duality and the uncertainty principle, etc. For the control groups, corresponding figure lay between 83 and $93 \%$.

We can conclude from these great differences between the experimental and control groups that the customary serious misunderstanding of modern physics (and, in general, of physics development) shown by pupils are not due to the special difficulty of modern physics but chiefly to incorrect teaching orientations. 
The results obtained allow us to put forward the following conclusions (which are in agreement with our initial hypotheses):

1. High school teachers and textbooks transmit an incorrect image of science, which ignores the existence of crises and paradigm shifts. The introduction of topics of modern physics, in particular, takes place without reference to its essential novelty or to the main differences between the classical and the new paradigm. A suitable occasion for showing the richness of the development of science and the importance of science revolutions is thus wasted.

2. As a result of this orientation, pupils develop a very confused view of the evolution of scientific knowledge and show serious misconceptions of modern physics topics.

3. It is relatively easy to give a more correct view of physics - and particularly of modern physics - with a constructivist approach in which programmes of activities are used that aim at producing in pupils a conceptual change similar to the historical change of paradigm.

\section{References}

Ausubel, D. P. (1968). Educational Psychology. A Cognitive View. New York, Holt, Rinehart \& Winston.

Driver, R, and Oldham, V. (1986). A constructivist approach to curriculum development in science. Studies in Science Education, 5, 105-122.

Garcia Casta.̃̃eda, M. (1985). An abuse with the wave properties of matter. American Journal of Physics, 53, 373-374. Gir., D. (1983). Tres paradigmas en la enseñanza de las ciencias. Enseñanza de las Ciencios, 1 ,
26-33.

GiL, D. and Carrascosa, J. (1985). Science learning as a conceptual and methodological change. European Jounal of Science Education, 7, 231-236.

GIL, I. and Carrascosa, J. (1990). What to do about science 'misconceptions'. Science Education, 74, 531-540.

Git., D., Carkascosa, J., Fi:kio, C. and Martinez, J. (1991). La enseñanza de las ciencias en la educatión secundaria Barcelona, Horsori.

HASHEWH, M. Z. (1986). 'Towards an explanation of conceptual change. Furopean Journal of Science Edtcation, 8, 229-249.

Hodson, D. (1985). Philosophy of science, science and science education. Studies in Science Education, 12, 25-27.

Honson, D. (1988). Toward a philosophically more valid science curriculum. Science Education, 72, 19-40.

Lringman, R. L. (1982). Confused physics: a tutorial critique. The Physics Teacher, 20, 519523.

McDermott, I. C. (1984). Critical review of research in the domain of mechanics. In G. Delacôte and A. Tiberghien (Eds.). Research on Physics Education. Paris, Editions de CRNS.

Mit.LAR, R. and Driver, R. (1987). Beyond processes. Studies in Science Education, 14, 33-62.

Posniek, G. S., Strike, K. A., Hewson, P. W. and Hertzog, W. A. (1982). Accommodation of a scientific conception: towards a theory of conceptual change. Science Education, 66, 211-227.

Solbes, J. (1986). La introducción de los conceptos básicos de Física moderna. $\mathrm{PhD}$ thesis, Universitat de València.

Solomon, J. (1987). Social influences on the construction of pupils' understanding of science. Studies in Science Education, 14, 63-82.

VienNot, L. (1976). Le raisonnement spontané en dynamique elémentaire. PhD thesis, Université Paris-7, France.

WARREN, J. W. (1976). The mystery of mass-energy. Physics Education, 11, 52- 54.

WheAtiey, G. H. (1991). Constructivist perspectives on science and mathematics learning. 